\title{
Manifestation of electron-electron interaction in the magnetoresistance of graphene
}

\author{
Johannes Jobst, ${ }^{1}$ Daniel Waldmann, ${ }^{1}$ Igor V. Gornyi,${ }^{2,3}$ Alexander D. Mirlin,,${ }^{2,4,5}$ and Heiko B. Weber ${ }^{1, \text { * }}$ \\ ${ }^{1}$ Lehrstuhl für Angewandte Physik, Universität Erlangen-Nürnberg, 91058 Erlangen, Germany \\ ${ }^{2}$ Institut für Nanotechnologie, Karlsruhe Institute of Technology, 76021 Karlsruhe, Germany \\ 3 A.F. Ioffe Physico-Technical Institute, 194021 St. Petersburg, Russia. \\ 4 Inst. für Theorie der kondensierten Materie, Karlsruhe Institute of Technology, 76128 Karlsruhe, Germany \\ 5 Petersburg Nuclear Physics Institute, 188300 St. Petersburg, Russia.
}

(Dated: October 11, 2018)

\begin{abstract}
We investigate the magnetotransport in large area graphene Hall bars epitaxially grown on silicon carbide. In the intermediate field regime between weak localization and Landau quantization the observed temperature-dependent parabolic magnetoresistivity (MR) is a manifestation of the electron-electron interaction (EEI). We can consistently describe the data with a model for diffusive (magneto)transport that also includes magnetic-field dependent effects originating from ballistic time scales. We find an excellent agreement between the experimentally observed temperature dependence of MR and the theory of EEI in the diffusive regime. We can further assign a temperaturedriven crossover to the reduction of the multiplet modes contributing to EEI from 7 to 3 due to intervalley scattering. In addition, we find a temperature independent ballistic contribution to the $\mathrm{MR}$ in classically strong magnetic fields.

PACS numbers:
\end{abstract}

Graphene is an outstanding electronic material with unique physical properties and with excellent prospects for technological applications [1 4]. The linear band structure and the chiral nature of charge carriers result in a rich variety of phenomena [5 9] which can be largely described within a model of non-interacting electrons. Evidence for electron-electron interaction (EEI) in graphene, however, is sparse. The most spectacular example is the occurrence of the fractional quantum Hall effect and the insulating phase in strong magnetic fields 10, 11]. Another line of research involves the investigation of EEI-induced renormalization of the Dirac spectrum in graphene [12, 13].

Furthermore EEI plays a prominent role in electron transport also in the absence of quantizing magnetic field, when the system is a Fermi liquid. In particular, EEI leads to a quantum contribution to zero-field resistivity, that in two dimensional electron gases (2DEG) at sufficiently low temperatures $T$ displays a logarithmic $T$ dependence 14]. Logarithmic corrections may further stem from weak localization (WL), when observed in very low fields. At somewhat higher fields logarithmic $\rho(T)$ data were interpreted as EEI 15 17] or Kondo effect [18]. This indicates that an evaluation of the temperature dependence $\rho(T)$ alone is insufficient for identification of the underlying mechanisms. A way to unambiguously single out the EEI effect is to study the temperature-dependent magnetoresistance (MR) of a 2DEG in non-quantizing magnetic fields, between weak fields where WL is operative and strong fields where Landau quantization is fully developed. The analysis of EEI effects in the magnetotransport, which have been widely disregarded, is the scope of this paper.

For this investigation large sample dimensions are mandatory, as in small flakes mesoscopic conductance fluctuations would add to the MR and the finite sample size sets a low-energy cut-off to the EEI. This is the case for most experiments using exfoliated graphene. Therefore we use epitaxial graphene on insulating silicon carbide $(\mathrm{SiC})$ which is available with excellent homogeneity on the wafer scale [19]. Our special choice is quasi freestanding monolayer graphene (QFMLG) 20, 21] which represents a hole-conducting graphene material with a charge carrier density of $n \approx 3 \cdot 10^{12} \mathrm{~cm}^{-2}$ and a mobility $\mu$ of approximately $2000 \mathrm{~cm}^{2} /$ Vs. In contrast to other epitaxial graphene materials $n$ and $\mu$ are more or less independent of temperature due to a reduced coupling to the substrate 21]. The chiral nature of charge carriers in QFMLG has been proven in previous experiments [22].

For the present study we prepared Hall bars by standard electron beam lithography [23] and measured the magnetoresistivity $\rho(B)$ in four-point geometry for various temperatures $T$ using low-frequency lock-in technique. For all $\rho(B, T)$ curves the magnetic field $B$ was swept from -8 to $8 \mathrm{~T}$ twice to confirm reproducibility. All measurements were performed under cryogenic vacuum in order to reduce the effect of adsorbates. In total we investigated four samples of different size with very similar findings as concerned the EEI effects [24]. In this paper we focus on one particular sample S1 (330 $\mu \mathrm{m}$ long and $50 \mu \mathrm{m}$ wide channel) which shows all observed phenomena in one consistent data set. Figure 1(a) shows the magnetoresistivity raw data. Obviously the Landau quantization regime is not yet reached (however, it has been observed in higher fields 22]). This indicates that the damping of Shubnikov-de Haas oscillations in the sample is dominated by smooth disorder, so that the transport relaxation time is longer than the quantum scattering time.

At weak fields the WL correction is clearly visible as a 
zero-field peak. We have characterized it carefully and can accurately describe the anomaly within the standard graphene model [8, 25]. The evaluation shown in Fig. 2(b) yields the intravalley scattering time responsible for the breaking of the effective time-reversal symmetry within a single valley $\tau_{\star}=0.075 \mathrm{ps}$ and the intervalley scattering time $\tau_{\mathrm{iv}}=1.1 \mathrm{ps}$, together with the phase relaxation time $\tau_{\varphi}$, which is strongly temperature dependent [cf. Fig. 2(c)] and decays with increasing $T$ roughly as $T^{-1}$ as expected for electron-electron scattering in the diffusive regime [8, 14]. The WL data are qualitatively consistent with findings in exfoliated graphene where the typical time scales are $\tau_{\text {iv }}=0.8 \ldots 2.5 \mathrm{ps}$ and $\tau_{\star}=0.05 \ldots 0.3 \mathrm{ps}[8,26]$. The deviation from the $T^{-1}$ dependence at low $T$ presumably stems from the presence of magnetic impurities [17].

At stronger fields we observe that the curves have roughly parabolic shape and vary with temperature, showing an evolution from negative to positive magnetoresistivity as the temperature rises. Thus, the observed $\ln T$ dependence (see the analysis below) indicates that we are dealing with the quantum contribution due to EEI as classical contributions to the magnetoresistance are temperature independent at low $T$. Another striking feature of Fig. 1(a) is a crossing point of low- $T$ curves that moves to a higher value of magnetic field at higher temperatures.

For further analysis the data are symmetrized with respect to $B$ and relative differences $\left(\rho-\rho_{0}\right) / \rho_{0}^{2} \approx-\delta \sigma$ are considered. We choose $\rho_{0}=\rho(0.5 \mathrm{~T})$ to eliminate the effect of the WL correction at $|B|<0.5 \mathrm{~T}$. The resulting curves $\left(\rho-\rho_{0}\right) / \rho_{0}^{2}(B)$ are plotted in Fig. 1(b). Again the transition from negative to positive magnetoresistance with rising temperature is visible. A closer look at intermediate temperatures reveals that the curvature differs in its sign even within the same curve between weaker $(|B|<4.5 \mathrm{~T})$ and stronger fields. Indeed for all curves two separate parabolas $\left(\omega_{\mathrm{c}} \tau_{\mathrm{tr}}\right)^{2} \frac{e^{2}}{2 \pi^{2} \hbar} \cdot M$ with different curvatures $M_{\mathrm{i}}$ and $M_{\mathrm{o}}$ have to be taken into account for the inner and the outer field region respectively, where $\omega_{\mathrm{c}}=\frac{v_{\mathrm{F}} e}{\hbar \sqrt{\pi n}} B$ is the cyclotron frequency. As a particular example Fig. 1(c) shows this decomposition for the lowest $(T=1.8 \mathrm{~K})$ and an intermediate $(T=20 \mathrm{~K})$ temperature. The transition field of $\approx 4.5 \mathrm{~T}$ is constant for all temperatures.

We analyze the data with the help of a model that successfully described the magnetoresistance in many 2DEGs in non-quantizing fields (see Refs [27] and 28] for review) and is equally suited for graphene at large dimensionless conductance. The model takes EEI into account and covers the crossover from the diffusive to the ballistic regime. The diffusive regime where $k_{\mathrm{B}} T \tau_{\mathrm{tr}} / \hbar \ll 1$ is observed for the lowest-temperature curves. The transport time $\tau_{\mathrm{tr}} \approx 0.045 \mathrm{ps}$ is derived from the Drude resistivity. The higher- $T$ curves correspond to the crossover regime,

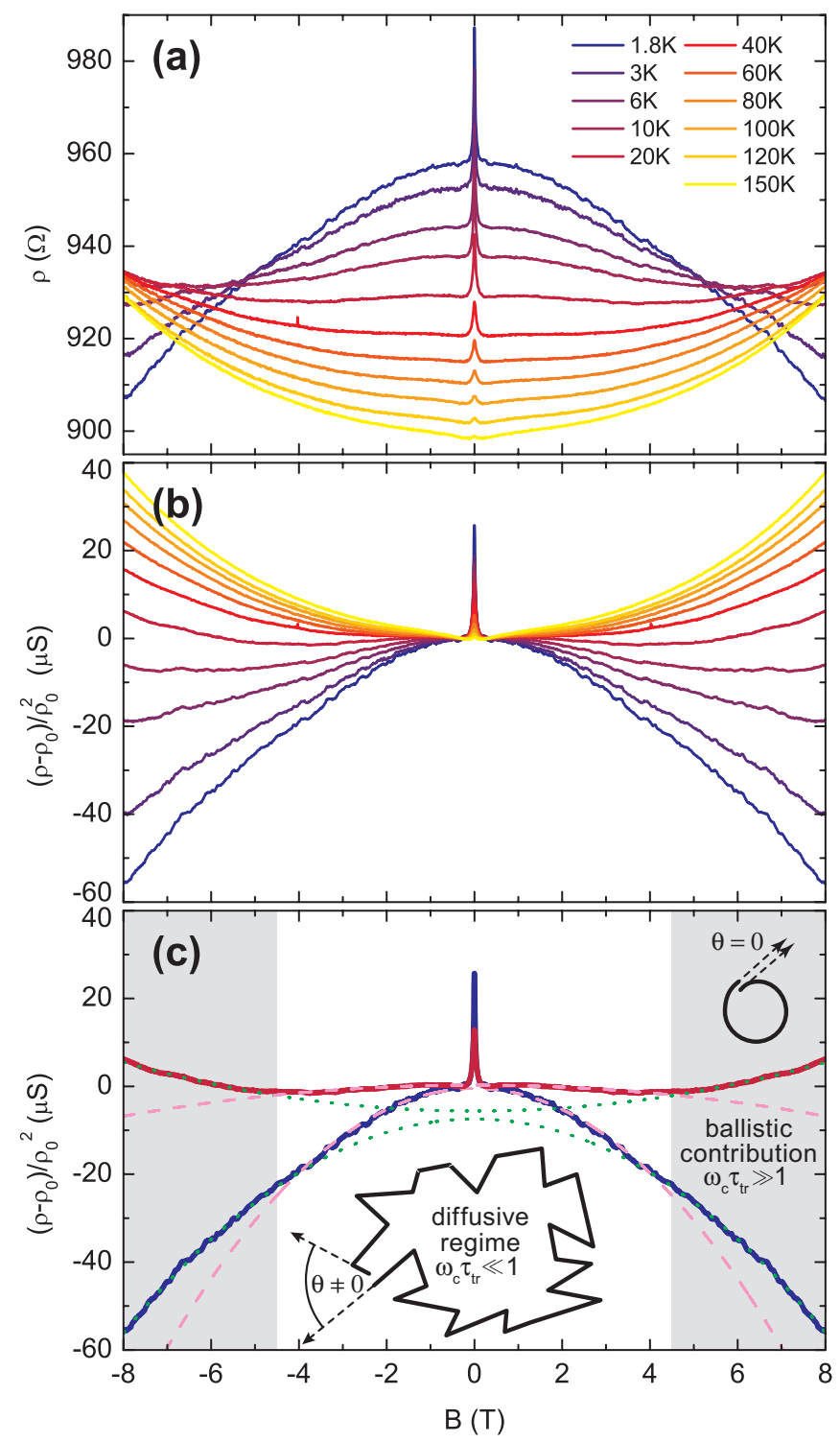

FIG. 1: Evolution of magnetoresistivity with temperature. (a) The curvature changes sign with increasing temperature. Further, for temperatures $T \leq 10 \mathrm{~K}$ the curves cross at $B \approx$ $5 \mathrm{~T}$; for higher temperatures the crossing point shifts towards a larger value $B \approx 9 \mathrm{~T}$. (b) Relative magnetoresistivity ( $\rho-$ $\left.\rho_{0}\right) / \rho_{0}^{2}$. (c) Two parabolas $\left(\omega_{\mathrm{c}} \tau_{\mathrm{tr}}\right)^{2} \frac{e^{2}}{2 \pi^{2} \hbar} \cdot M$ are fitted to the data in (b) (shown representative for $1.8 \mathrm{~K}$ and $20 \mathrm{~K}$ ): one in the diffusive regime for weak fields $1 \mathrm{~T}<|B|<4.5 \mathrm{~T}$ (dashed) and one in the diffusive regime with ballistic contributions for $|B|>5 \mathrm{~T}$ (dotted). Two sketches illustrate the different scattering mechanisms in both regimes.

where the importance of ballistic effects [27, 29 31] increases. Still, for all studied temperatures and samples the condition $k_{\mathrm{B}} T \tau_{\mathrm{tr}} / \hbar \lesssim 1$ is fulfilled which means that the ballistic regime is not reached.

In the diffusive regime, EEI induces a logarithmicin- $T, B$-independent correction $\delta \sigma_{\text {ee }}$ to the longitudinal conductivity, while not affecting the Hall conductivity 
[14]. As a result, EEI gives rise to a magnetoresistance $\delta \rho / \rho_{0} \propto(\mu B)^{2} \delta \sigma_{\text {ee }} / \sigma_{0}$ (here $\sigma_{0}$ is the zero- $B$ Drude conductivity) which is parabolic in $B$ [32, 33]:

$$
\rho=\rho_{0}+\left[\left(\omega_{\mathrm{c}} \tau_{\mathrm{tr}}\right)^{2}-1\right] \frac{e^{2} \rho_{0}^{2}}{2 \pi^{2} \hbar}\left[A \cdot \ln \left(\frac{k_{\mathrm{B}} T \tau_{\mathrm{tr}}}{\hbar}\right)\right],
$$

This correction includes two contributions: the Coulomb term and the Hartree term. The coefficient $A$ is the sum of these two terms

$$
A=A_{\mathrm{C}}+A_{\mathrm{H}}=1+c\left[1-\frac{\ln \left(1+F_{0}^{\sigma}\right)}{F_{0}^{\sigma}}\right],
$$

with the Fermi liquid constant $F_{0}^{\sigma}$. It is a measure for the interaction strength and in the case of weak interaction it can be calculated within Thomas-Fermi approximation by integrating the interaction matrix element over all scattering angles [16]:

$$
F_{0, \text { theo }}^{\sigma}=-\alpha \int \frac{\cos ^{2} \theta / 2}{2 \pi(\sin \theta / 2+2 \alpha)} \mathrm{d} \theta,
$$

with the interaction constant $\alpha=e_{*}^{2} /\left(4 \pi \varepsilon_{0} \hbar v_{\mathrm{F}}\right) \approx 0.41$ and an effective charge $e_{*}=e \sqrt{2 /(\varepsilon+1)} \approx 0.43 e$ taking into account the screening of Coulomb interaction by the $\mathrm{SiC}$ substrate $\left(\varepsilon_{\mathrm{SiC}}=9.66\right)$. For the given case equations (2) and (3) yield $A\left(F_{0 \text {, theo }}^{\sigma}\right) \approx 1-0.046 \cdot c$ and $F_{0 \text {, theo }}^{\sigma} \approx-0.09$ respectively. The constant $c$ is the number of multiplet channels participating to EEI.

Here the peculiarity of graphene enters at the following two points. First, the $\cos ^{2} \theta / 2$ reflects the influence of chirality on scattering of charge carriers in graphene. Second, the channel statistics of graphene is different from a standard 2DEG and depends on the interplay of symmetry-breaking time scales. In our samples the hierarchy is $\tau_{\text {tr }}<\tau_{\star}<\tau_{\text {iv }}$ as extracted from the analysis of the WL peak. This hierarchy is also found in exfoliated graphene [8]. In perfect graphene the spin and valley degeneracy gives rise to 15 multiplet channels. For temperatures $T<\hbar / k_{\mathrm{B}} \tau_{\star}$ the breaking of the time-reversal symmetry within a single valley (due to, for example, the trigonal warping or ripples) suppresses 8 channels out of 15, yielding $c=7$ [16]. The intervalley scattering further reduces the number of multiplet channels to $c=3$ (spin-triplet). This standard 2DEG case is expected for sufficiently low temperatures $T<T_{\mathrm{iv}}:=\hbar / k_{\mathrm{B}} \tau_{\mathrm{iv}} \approx 7 \mathrm{~K}$.

When analyzing the prefactors $A$ of formula (1), one can expect to see the predicted crossover between $c=3$ and $c=7$ in the temperature dependence of the curvature of the magnetoresistivity. Figure 2 plots the curvatures $M_{\mathrm{i}}$ for the inner (circles) and $M_{\mathrm{o}}$ for the outer (squares) field region as a function of temperature (more explicitly $\left.k_{\mathrm{B}} T \tau_{\mathrm{tr}} / \hbar\right)$. In this plot two regions of different slope can be distinguished, with a crossover at $T_{\mathrm{iv}} \approx 7 \mathrm{~K}$, that perfectly fits the temperature scale given by $\tau_{\text {iv }}$ (extracted from WL data). From Eq. (1) it is obvious that the slope of the curvatures $M$ in this plot over $k_{\mathrm{B}} T \tau_{\operatorname{tr}} / \hbar$

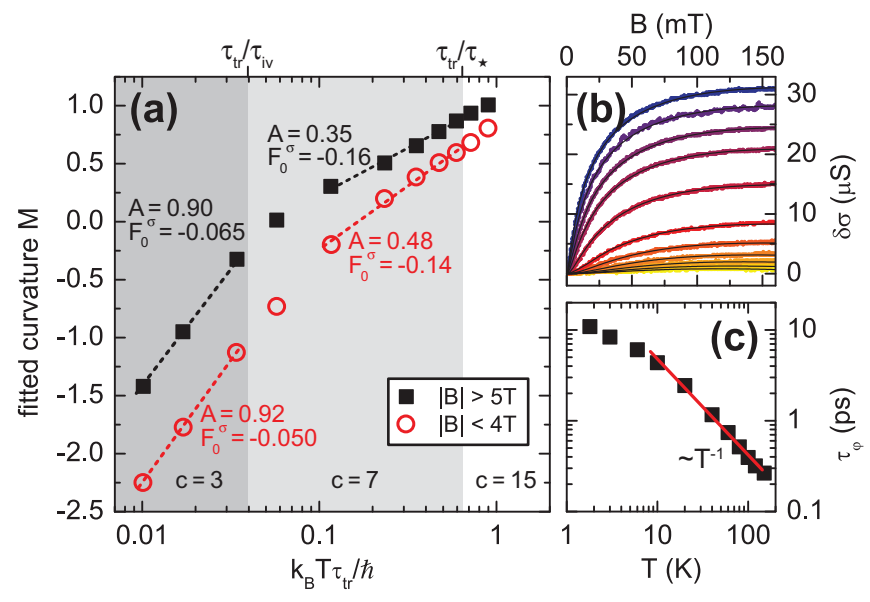

FIG. 2: (a) The curvature $M$ of the parabolic MR extracted from the fits to the curves shown in Fig. 1(b) at weak (circles) and strong (squares) magnetic field. A kink is observed at $T=\hbar / \tau_{\mathrm{iv}} k_{\mathrm{B}} \approx 7 \mathrm{~K}$ as the number of contributing multiplet channels changes from $c=3$ to $c=7$. From the slopes $A$ the Fermi-liquid constant $F_{0}^{\sigma}$ is calculated for the different regions. (b) Weak localization correction to the conductivity at weak fields. Straight lines are fits of the graphene-specific model 25] that allows to determine the scattering times $\tau_{\star}$, $\tau_{\text {iv }}$ and the phase-coherence time $\tau_{\varphi}$. (c) As expected for electron-electron scattering in the diffusive regime $\tau_{\varphi}$ decays as $T^{-1}$.

(on logarithmic scale) is equal to the coefficient $A$. Hence the change in slope is caused by a different number of contributing multiplet channels in the two regimes. Using $c=3$ as expected for $T<T_{\mathrm{iv}}$ a Fermi liquid constant of $F_{0, \mathrm{i}}^{\sigma}=-0.05$ and $F_{0, \mathrm{o}}^{\sigma}=-0.06$ can be extracted for the inner and outer field region respectively. For $T>T_{\mathrm{iv}}$ $F_{0, \mathrm{i}}^{\sigma}=-0.14$ and $F_{0, \mathrm{o}}^{\sigma}=-0.16$ are found with $c=7$. The obtained values are reasonably close to the value of the theoretically expected $F_{0 \text {,theo }}^{\sigma}=-0.09$. The higher values at higher temperatures can qualitatively be attributed to the crossover towards ballistics (see discussion below).

It is worthwhile to compare the extracted values of $F_{0}^{\sigma}$ to those measured in a $2 \mathrm{D}$ electron gas in conventional semiconductor structures. The main factor determining the Fermi liquid constant is the widely used parameter $r_{\mathrm{s}}$ controlling the ratio of characteristic potential and kinetic energies. It depends on the electron charge, the dielectric constant, and the Fermi velocity (in our case $r_{\mathrm{s}}=\sqrt{2} \alpha$ ). The central difference to semiconductors is that there the Fermi velocity depends strongly on the electron concentration $n$, so that $r_{\mathrm{s}}$ varies substantially (typically in the range from 2 to 20, leading to a change of $F_{0}^{\sigma}$ between 0.15 and 0.7 ; see, e.g., Ref. [34]). In contrast, in graphene the velocity is roughly $n$-independent, and $r_{\mathrm{s}}$ remains relatively small. In addition, for given $r_{\mathrm{s}}$ the Fermi-liquid constant is somewhat suppressed in graphene due to chiral character of carriers, see Eq. (3). 
A hallmark of the diffusive regime is that the curvature of the MR correlates with the sign and amplitude of the correction to the zero- $B$ resistivity. In particular this leads to the well defined crossing point (CP) in Fig. [1(a). At this point at $B_{\mathrm{CP}}=5.2 \mathrm{~T}$ [Fig. 3(a)] where $T$ dependent and $B$-dependent corrections exactly cancel, the resistivity has the classical Drude value [35, 36]. The value of this $\mathrm{CP}$ agrees well with $B_{\mathrm{CP}}=4.8 \mathrm{~T}$ expected from $\omega_{\mathrm{c}} \tau_{\mathrm{tr}}=1$. The overall consistency of our findings (together with the emergence of the crossing point) confirms that the applied diffusive model describes the physics properly and hence is a reliable way to separate the effect of EEI from other corrections.

Now we turn to the observations which are beyond the purely diffusive model described by Eq. (1). (I) First, we find different curvatures of the MR for weak and strong magnetic fields [cf. Fig. 1(c)]. (II) Second, the higher-T MR curves have a tendency to form an apparent second crossing point [cf. Fig. [3(b)]. These two observations suggest that the diffusive mechanism, which governs the logarithmic temperature dependence of the curvature of the $\mathrm{MR}$ in the whole range of magnetic fields and temperatures explored in this work, is supplemented by ballistic effects that become more pronounced at higher $T$ and magnetic field.

Observation (I) implies that the MR contains a $T$ independent contribution which stems from ballistic scales [27, 31] and is sensitive to the relation between $\omega_{\mathrm{c}}$ and $\tau_{\mathrm{tr}}$. As the magnetic field increases, for $B>1 / \mu$ (which corresponds to $\omega_{\mathrm{c}} \tau_{\mathrm{tr}}>1$ ) electrons can accomplish closed cyclotron orbits which leads to an enhanced probability of scattering angles $\theta \approx 0$ [sketch in Fig. [1(c)]. This correlation causes a change of the strength of EEI [see Eq. (3)] in the ballistic contribution and hence the overall curvature of the MR. In addition, there might be a temperature-independent contribution due to a classical mechanism of magnetoresistance (due to non-Markovian processes, for review see Ref. [28]) operative in classically strong magnetic fields $B>1 / \mu$ that leads to a vertical shift between the values of $M_{\mathrm{i}}$ and $M_{\mathrm{O}}$.

Observation (II) indicates that the $T$ dependence of the zero- $B$ resistivity $\rho_{0}$ does not fully determine the curvature of the MR [27, 31, 36, 37]. This signals the onset of a crossover to the ballistic regime at $T \geq 20 \mathrm{~K}$. Indeed, at $k_{\mathrm{B}} T \tau_{\mathrm{tr}} / \hbar<1$ the $T$ dependence of $\rho_{0}$ stems from the combination of a logarithmic diffusive correction and a $T$-dependent correction $\delta \mu(T)$ to the mobility (Ballistic return processes may give rise to ballistic $T$ dependent corrections to the mobility [38]). At lowest temperatures, the logarithmic diffusive correction dominates. With increasing $T$, the diffusive term decreases while $\delta \mu(T)$ grows. Importantly, the processes giving rise to a $T$-dependent correction to the mobility do not lead to the MR at finite $B$, in contrast to the logarithmic diffusive term. Therefore, $\delta \mu(T)$ only shifts all resistivity curves $\rho(B)$ in the vertical direction (in the crossover

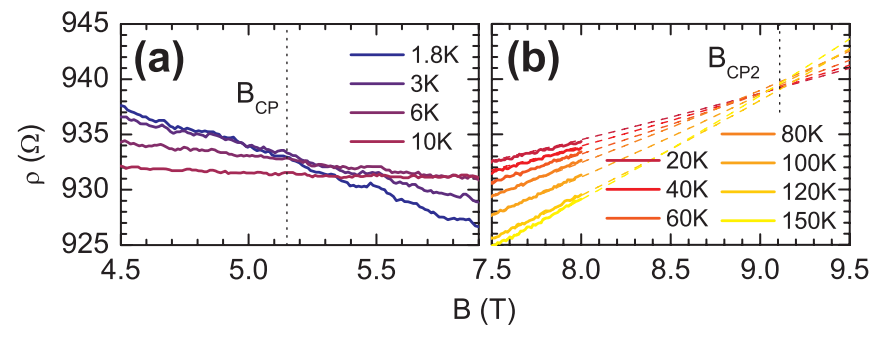

FIG. 3: Zoom on the crossing points. (a) The crossing point at $B_{\mathrm{CP}}=5.2 \mathrm{~T}$ is already visible in Fig. 1. (b) For higher temperatures, an apparent crossing point at $B_{\mathrm{CP} 2} \simeq 9.1 \mathrm{~T}$ emerges by extrapolating (dashed lines) the parabolic shape of the curves onto $B>8 \mathrm{~T}$.

to the ballistic regime the diffusive and ballistic contributions to this shift become comparable), while the $T$ dependence of the MR curvature is still given solely by the logarithmic term. This explains the shift of the $\mathrm{CP}$ towards an apparent high- $T \mathrm{CP}$ at $B_{\mathrm{CP} 2} \simeq 9.1 \mathrm{~T}$ that emerges when the MR parabolas are extrapolated to stronger fields [Fig. [3(b)]. Its observation thus proves that ballistic corrections to $\rho_{0}$ gain importance at higher temperatures.

Theoretical results for $\mathrm{MR}$ in the ballistic (and crossover) regimes depend on the microscopic model of disorder 27, 28]. Therefore, a systematic study of MR in the crossover and ballistic regimes may be helpful for clarifying the character of disorder in the system (which is particularly important for electron transport in graphene [39]); we relegate this to a future work. In this paper, we restrict ourselves to applying the diffusive model supplemented at strong $B$ by a $T$-independent ballistic contribution, which is a reasonably good approximation in the considered temperature range.

In conclusion, we have studied the magnetotransport in graphene epitaxially grown on silicon carbide. We observe a temperature-dependent parabolic magnetoresistivity which is a manifestation of the EEI. The temperature dependence of the MR curvature is logarithmic, as expected for the diffusive regime. We find a pronounced kink in this dependence, that is related to the reduction of the number of multiplet modes contributing to EEI from 7 to 3 due to intervalley scattering.

We show that our model of diffusive transport with ballistic contributions can describe the underlying physics properly. The analysis of the temperature dependence of the magnetoresistance allows for an accurate description of the EEI interaction correction in the diffusive regime. At magnetic fields higher than the observed crossing point $\left(\omega_{\mathrm{c}} \tau_{t r}>1\right)$ the magnetoresistance shows an additional (compared to weak field) $T$-independent parabolic contribution due to ballistic effects. Further quantitative analysis of the crossover and ballistic regimes should be helpful for shedding light on the nature of disorder in graphene structures. 
Our work demonstrates that the analysis of MR in the intermediate range of $B$ is a convenient tool for exploring many-body effects in graphene. In particular, this method allows to precisely distinguish the effect of EEI from additional corrections to the temperature dependent resistivity that also have logarithmic temperature dependence. Furthermore, our approach allowed us to determine the Coulomb interaction strength in our samples with a rather high accuracy. The value of the interaction constant obtained from the fit to the experimental data is $F_{0}^{\sigma} \approx-0.06$ at low $T$ and -0.15 at high $T$ (where the system approaches the ballistic regime), which is close to the theoretical estimate for graphene on silicon carbide, $F_{0}^{\sigma} \approx-0.09$. These findings should also directly apply to other graphene samples (e.g., to exfoliated graphene) provided the system sizes are sufficiently large.

We gratefully acknowledge support within the Cluster of Excellence Engineering of Advanced Materials (www.eam.uni-erlangen.de), DFG Center for Functional Nanostructures, and DFG SPP 1459 "Graphene". We acknowledge the use of the hydrogen intercalation furnace provided by Th. Seyller.

* Electronic address: heiko.weber@physik.uni-erlangen.de URL: http://www.lap.physik.uni-erlangen.de/

[1] A. K. Geim, Rev. Mod. Phys. 83, 851 (2011).

[2] K. S. Novoselov, Rev. Mod. Phys. 83, 837 (2011).

[3] Y. Lin, C. Dimitrakopoulos, K. Jenkins, D. Farmer, H. Chiu, A. Grill, and P. Avouris, Science 327, 662 (2010).

[4] S. Bae, H. Kim, Y. Lee, X. Xu, J. S. Park, Y. Zheng, J. Balakrishnan, T. Lei, H. R. Kim, Y. I. Song, et al., Nature Nanotech. 5, 574 (2010).

[5] K. S. Novoselov, A. K. Geim, S. V. Morozov, D. Jiang, M. I. Katsnelson, I. V. Grigorieva, S. V. Dubonos, and A. Firsov, Nature 438, 197 (2005).

[6] Y. Zhang, Y. Tan, H. Stormer, and P. Kim, Nature 438, 201 (2005).

[7] N. Stander, B. Huard, and D. Goldhaber-Gordon, Phys. Rev. Lett. 102, 026807 (2009).

[8] F. V. Tikhonenko, A. A. Kozikov, A. K. Savchenko, and R. V. Gorbachev, Phys. Rev. Lett. 103, 226801 (2009).

[9] A. H. Castro Neto, F. Guinea, N. M. R. Peres, K. S. Novoselov, and A. K. Geim, Rev. Mod. Phys. 81, 109 (2009).

[10] X. Du, I. Skachko, F. Duerr, A. Luican, and E. Andrei, Nature 462, 192 (2009).

[11] K. Bolotin, F. Ghahari, M. Shulman, H. Stormer, and P. Kim, Nature 462, 196 (2009).

[12] D. C. Elias, R. V. Gorbachev, A. S. Mayorov, S. V. Morozov, A. A. Zhukov, P. Blake, L. A. Ponomarenko, I. V. Grigorieva, K. S. Novoselov, F. Guinea, et al., Nat. Phys. 7, 701 (2011).

[13] D. A. Siegel, C.-H. Park, C. Hwang, J. Deslippeand, A. V. Fedorov, S. G. Louie, and A. Lanzara, PNAS 108, 11365 (2011).
[14] B. L. Altshuler and A. G. Aronov, Electron-electron interactions in disordered conductors in Electron-Electron Interactions in Disordered Systems (North-Holland, Amsterdam, 1985).

[15] B. Jouault, B. Jabakhanji, N. Camara, W. Desrat, C. Consejo, and J. Camassel, Phys. Rev. B 83, 195417 (2011).

[16] A. A. Kozikov, A. K. Savchenko, B. N. Narozhny, and A. V. Shytov, Phys. Rev. B 82, 075424 (2010).

[17] S. Lara-Avila, A. Tzalenchuk, S. Kubatkin, R. Yakimova, T. J. B. M. Janssen, K. Cedergren, T. Bergsten, and V. Fal'ko, Phys. Rev. Lett. 107, 166602 (2011).

[18] J. H. Chen, L. Li, W. G. Cullen, E. D. Williams, and M. S. Fuhrer, Nature Phys. (2011).

[19] K. Emtsev, A. Bostwick, K. Horn, J. Jobst, G. L. Kellogg, L. Ley, J. McChesney, T. Ohta, S. A. Reshanov, J. Röhrl, et al., Nature Mater. 8, 203 (2009).

[20] C. Riedl, C. Coletti, T. Iwasaki, A. A. Zakharov, and U. Starke, Phys. Rev. Lett. 103, 246804 (2009).

[21] F. Speck, J. Jobst, F. Fromm, M. Ostler, D. Waldmann, M. Hundhausen, H. B. Weber, and T. Seyller, Appl. Phys. Lett. (2011).

[22] D. Waldmann, J. Jobst, F. Speck, T. Seyller, M. Krieger, and H. B. Weber, Nature Mater. 10, 357 (2011).

[23] J. Jobst, D. Waldmann, F. Speck, R. Hirner, D. K. Maude, T. Seyller, and H. B. Weber, Phys. Rev. B 81, 195434 (2010).

[24] See Supplemental Material at [URL will be inserted by publisher] for the results of samples S2, S3 and $S_{4}$.

[25] E. McCann, K. Kechedzhi, V. I. Fal'ko, H. Suzuura, T. Ando, and B. L. Altshuler, Phys. Rev. Lett. 97, 146805 (2006).

[26] D. W. Horsell, A. K. Savchenko, F. V. Tikhonenko, K. Kechedzhi, I. V. Lerner, and V. I. Fal'ko, Solid State Commun. 149, 1041 (2009).

[27] I. V. Gornyi and A. D. Mirlin, Phys. Rev. B 69, 045313 (2004).

[28] I. A. Dmitriev, F. Evers, I. V. Gornyi, A. D. Mirlin, D. G. Polyakov, and P. Wölfle, Phys. Status Solidi (b) 245, 239 (2008).

[29] G. Zala, B. N. Narozhny, and I. L. Aleiner, Phys. Rev. B 64, 214204 (2001).

[30] G. Zala, B. Narozhny, and I. Aleiner, Phys. Rev. B 64, 201201 (2001).

[31] I. V. Gornyi and A. D. Mirlin, Phys. Rev. Lett. 90, 076801 (2003).

[32] A. Houghton, J. R. Senna, and S. C. Ying, Phys. Rev. B 25, 2196 (1982).

[33] S. M. Girvin, M. Jonson, and P. A. Lee, Phys. Rev. B 26, 1651 (1982).

[34] E. A. Galaktionov, A. K. Savchenko, S. S. Safonov, Y. Y. Proskuryakov, L. Li, M. Pepper, M. Y. Simmons, D. A. Ritchie, E. H. Linfield, and Z. D. Kvon, Interactions in high-mobility 2D electron and hole systems in Fundamental Problems of Mesoscopic Physics (Kluwer Academic Publishers, Dorndrecht, 2004).

[35] G. M. Minkov, O. E. Rut, A. V. Germanenko, A. A. Sherstobitov, V. I. Shashkin, O. I. Khrykin, and B. N. Zvonkov, Phys. Rev. B 67, 205306 (2003).

[36] V. T. Renard, I. V. Gornyi, O. A. Tkachenko, V. A. Tkachenko, Z. D. Kvon, E. B. Olshanetsky, A. I. Toropov, and J.-C. Portal, Phys. Rev. B 72, 075313 (2005).

[37] L. Li, Y. Y. Proskuryakov, A. K. Savchenko, E. H. Linfield, and D. A. Ritchie, Phys. Rev. Lett. 90, 076802 
(2003).

[38] V. V. Cheianov and V. I. Fal'ko, Phys. Rev. Lett. 97, 226801 (2006).

[39] P. M. Ostrovsky, I. V. Gornyi, and A. D. Mirlin, Phys. Rev. B 74, 235443 (2006).

[40] S. Tanabe, Y. Sekine, H. Kageshima, M. Nagase, and H. Hibino, Physical Review B 84, 1 (2011). 


\section{Supplemental Material for 'Manifestation of electron-electron interaction in the magnetoresistance of graphene'}

This supplemental material is dedicated to elucidate the generality and reproducibility of the findings reported in the main text. We have observed the parabolic shape of the magnetoresistance (MR) in between the weaklocalization (WL) regime and the Landau quantization regime and its logarithmic temperature dependence for many samples. We have investigated it thoroughly for four samples. We decided to show data of sample S1 as one consistent data set in the main text. The data of the remaining three samples (S2, S3 and S4) are subject to this supplemental material. The quasi free-standing monolayer graphene (QFMLG) sample S2 had smaller size than $\mathrm{S} 1$ which made the analysis of the low temperature behavior difficult and increased the amplitude of the temperature-independent quasiclassical MR. The monolayer samples S3 and S4 (both not QFMLG) showed a large phonon contribution to the MR in view of a strong coupling to the substrate. Therefore, sample S1 was best suited for our analysis of electron-electron interaction (EEI) effects. Nevertheless, samples S2, S3, and S4 show the EEI effects in the temperature dependence of the MR quantitatively consistent with those in sample S1 (details are presented below).

\section{Similar sample type}

Figure 4 displays results of QFMLG sample S2 that is similar to sample $\mathrm{S} 1$ presented in the main text. The main difference is that $\mathrm{S} 2$ is smaller $(10 \mu \mathrm{m}$ wide, $70 \mu \mathrm{m}$ long) which made it difficult to analyze the low temperature behavior and increased the amplitude of the $T$ independent quasiclassical MR. In particular at lowest temperatures universal conductance fluctuations become apparent. Along with the somewhat lower mobility, the crossing point has moved to higher fields, and a field driven cross-over can not be detected. The evaluation of the curvature of the MR, however, shows the same crossover from $c=3$ to $c=7$ with rising temperature. The analysis yields similar values of the Fermi-liquid constant $F_{0}^{\sigma}$, at least for high temperatures. At low temperatures the data quality and density is unfortunately not as good as for sample S1 and therefore does not allow to extract $F_{0}^{\sigma}$ for the $c=3$ region.

\section{Different epitaxial graphene material}

Beyond QFMLG samples S1 and S2 presented in the main text and above, we investigated two samples $\mathrm{S} 3$ and
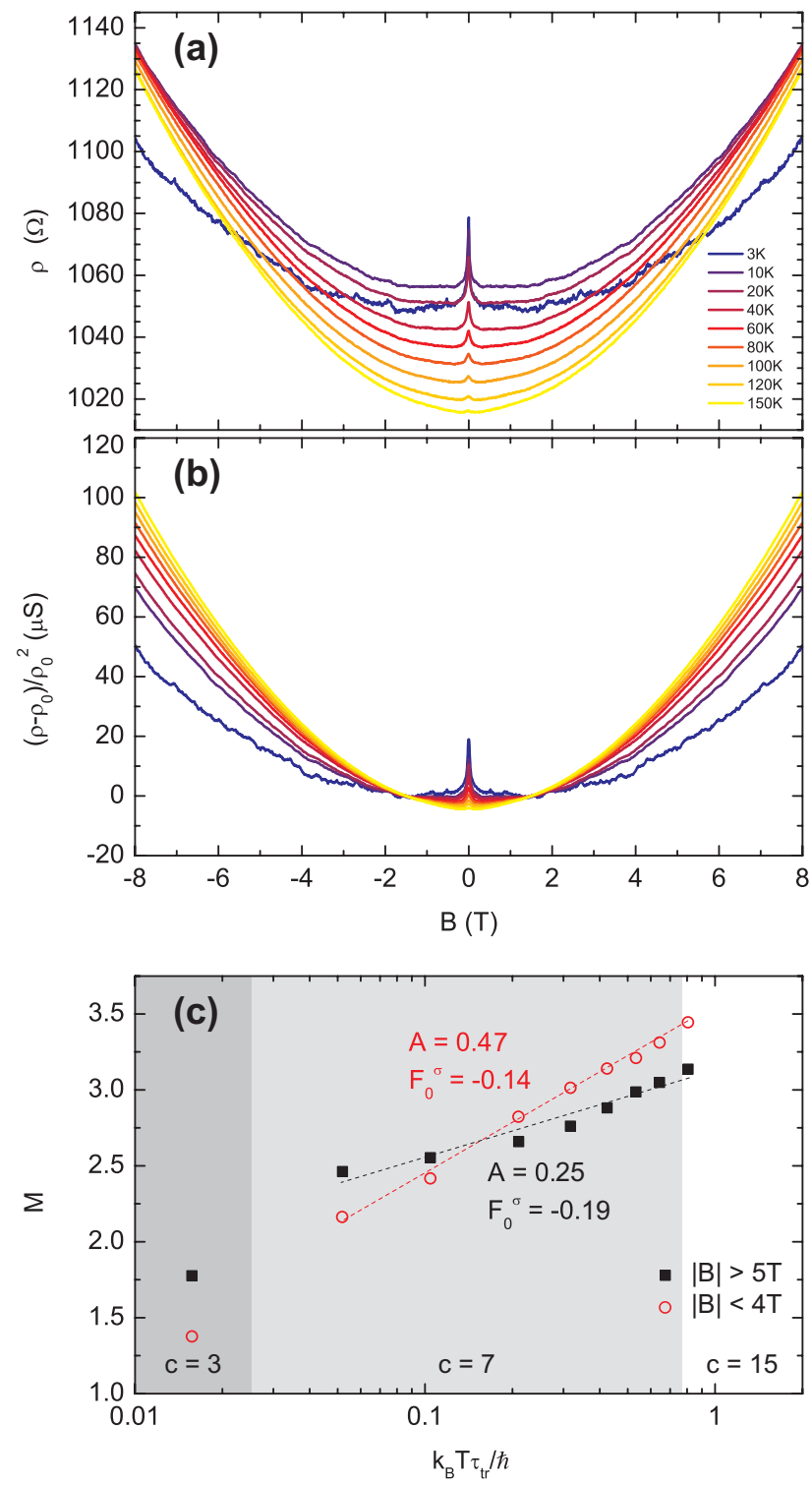

FIG. 4: (a) The magnetoresistivity raw data exhibits a parabolic shape and an apparent crossing point at $\approx 9 \mathrm{~T}$. (b) The relative magnetoresistivity shows the evolution of the curvatures with temperature. (c) Evaluation of the curvatures $M$ yields the Fermi-liquid constant. Intervalley scattering time $\tau_{i v}=1.5 \mathrm{ps}$ and intravalley scattering time $\tau_{\star}=0.051 \mathrm{ps}$ were extracted from WL data.

S4 using monolayer graphene (MLG) 19]. Microscopically the materials differ in an interface layer underneath the graphene 20, 21]. Phenomenologically, they differ in the sign of charge carriers (holes for QFMLG and electrons for MLG), and in different coupling to substrate phonons [40]. As a consequence of the latter, MLG has a substantial dependence of the resistivity $\rho$ on temperature $T$. It can readily be seen [Fig. 5 (a)] that a strong temperature dependent offset of the MR curves result. Therefore the occurrence of a crossing point is obscured. Figures 5 (b) and (d) show the further analysis carried out in exactly the same manner as presented in the main text. 

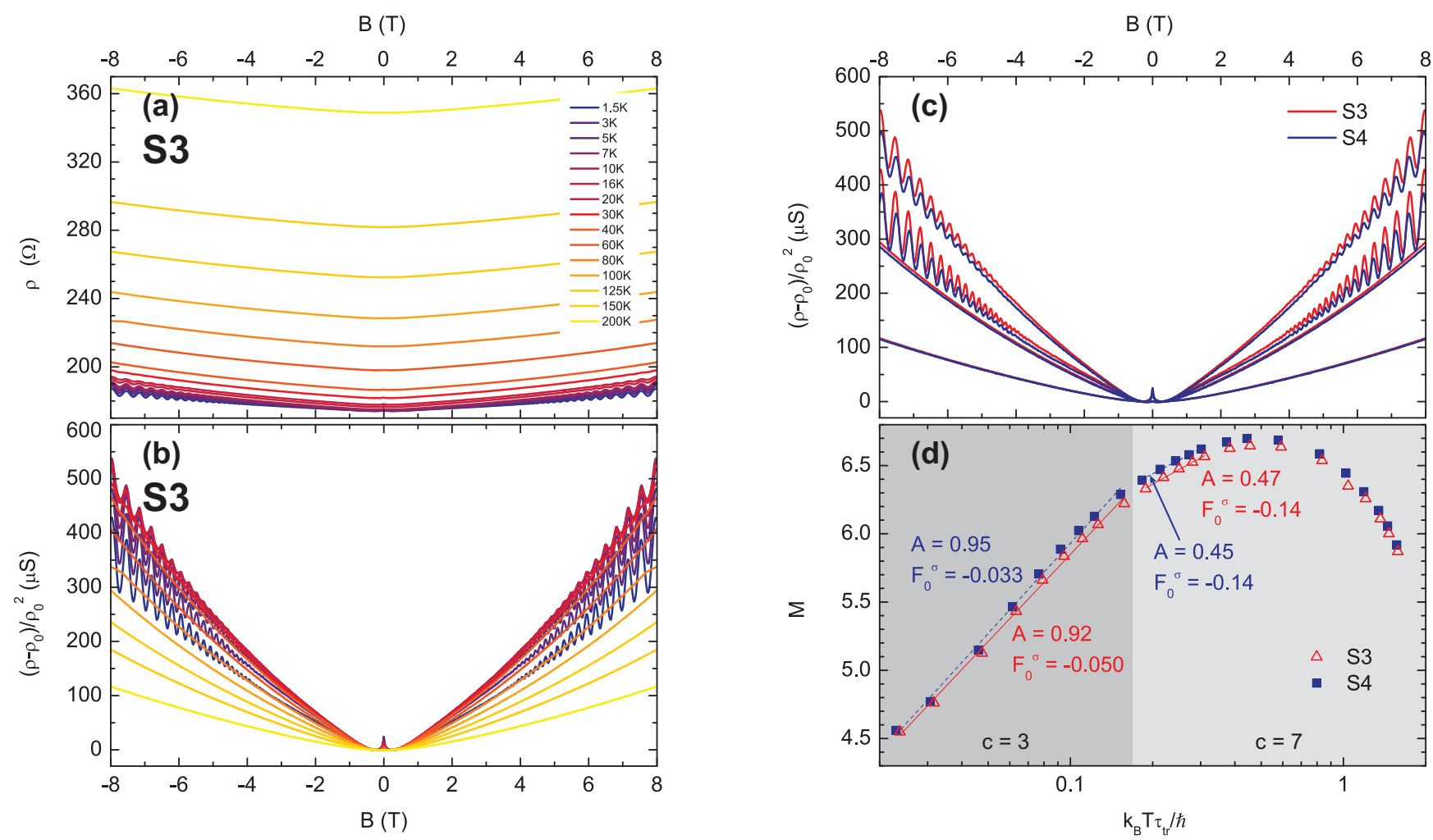

FIG. 5: The magnetoresistivity raw data of sample S3 (a) and S4 shows a pronounced vertical shift due to the coupling to substrate phonons in addition to the parabolic MR. At low temperatures and high fields Shubnikov-de Haas oscillations are visible. The relative magnetoresistivity (b) displays the evolution of the MR curvature with temperature. As MLG samples S3 and S4 are processed on the same chip their electronic properties are virtually identical as illustrated in (c) where MR curves for $10 \mathrm{~K}, 1.5 \mathrm{~K}, 100 \mathrm{~K}$ and $200 \mathrm{~K}$ (from top to bottom) are compared. Thus, also the evaluation of the MR curvatures $M$ of samples S3 and S4 are nearly equal (d). Note that the extracted values are very similar to the findings on sample S1. The scattering times $\tau_{i v}=0.53 \mathrm{ps}$ and $\tau_{\star}=0.025$ ps were extracted from WL data.

The enhanced mobility has two main consequences: (i) quantum oscillations occur at substantially lower fields as in S1. (ii) the obscured cross-over point also moves to lower fields and therefore the field range of the inner parabola (between WL regime and the obscured crossingpoint) is compressed. Hence it is difficult to evaluate the inner curvature $M_{i}$ and we restrict ourselves to $M_{o}$. The evaluation of the Fermi-liquid constant results in excellent agreement with the values extracted for S1. This displays the robustness of the findings taking into account that a different graphene material, a different charge carrier density and a different charge carrier type is present. The downturn in Fig. 囵(d) happens close to $k_{B} T \tau_{t r} / \hbar=1$ where the diffusive picture becomes inadequate. In addition, the phonon effects at high temperatures may affect the $T$ dependence of the MR curvature.

\section{Reproducibility}

One advantage of epitaxial graphene is the ability to very well reproduce data. As an example we show the MR of two samples S3 and S4 with nominally identical geometry ( $50 \mu \mathrm{m}$ wide, $330 \mu \mathrm{m}$ long) being processed on the same chip. Differences can hardly be recognized [Fig. [5(c) and (d)]. The above examples, however, show that when different fabrication parameters or different processing are chosen, the resulting samples may differ, most evidently in mobility and/or charge density. 